•物种概念专题・

\title{
菌物分类学研究中常见的物种概念
}

\author{
姚一建 ${ }^{*}$ 李 熠 ${ }^{1,2}$ \\ 1 (中国科学院微生物研究所真菌学国家重点实验室, 北京 100101) \\ 2 (福建农林大学植物保护学院植物病毒研究所福建省植物病毒学重点实验室, 福州 350002)
}

\begin{abstract}
摘要: 物种是生物多样性与分类学研究的基本单元, 物种识别是生物学研究的基本问题之一。物种的划分一直以 来都没有一个明确统一的标准, 这使得分类学多少带有主观的色彩, 并经常被看作艺术而不完全是科学的研究。 本文简要概述了菌物分类学研究中常见的 3 个物种概念, 即形态学种、生物学种和系统发育学种的背景和应用现 状, 并通过实例讨论了这 3 个物种概念的特点及应用中存在的问题, 特别是各个物种概念之间的交错, 以期为菌物 分类学研究和物种概念探讨提供参考。
\end{abstract}

关键词: 物种; 物种概念; 物种划分; 菌物

\section{Species concepts commonly used in fungal taxonomy}

\author{
Yijian $\mathrm{Yao}^{1 *}$, Yi $\mathrm{Li}^{1,2}$ \\ 1 State Key Laboratory of Mycology, Institute of Microbiology, Chinese Academy of Sciences, Beijing 100101 \\ 2 Fujian Province Key Laboratory of Plant Virology, Institute of Plant Virology, College of Plant Protection, Fujian Ag- \\ ricultural and Forestry University, Fuzhou 350002
}

\begin{abstract}
Both species concept and recognition are fundamental topics in the studies of taxonomy and biodiversity, and also in biological researches. There has never been any unanimous and clear standard for species dilimitation and, thus, taxonomy has more or less been regarded as subjective and often seen as art rather than scientific research. The backgrounds and the current status of three commonly used species concepts, i.e. morphological, biological and phylogenetic species concepts, in the research of fungal taxonomy are briefly reviewed here. The application of the three species concepts are discussed with examples in fungal taxonomy, especially on interlaces and incongruences between the concepts. The materials presented here may provide some useful information for the study on taxonomy and the investigation on species concept.
\end{abstract}

Key words: species; species concept; species separation; fungi

物种的划分是物种多样性研究、物种保护和利 用的基础(洪德元，2016a，b)，也是生物分类学研究 的主要内容。狭义的分类学仅包括物种的划分、鉴 定和归类，而广义的分类学还探讨物种的相互关系 及系统学, 往往还包含了系统发育和进化的内容。 在现代生物分类学早期(Linneaus, 1753), 物种被认 为是上帝创造的，其特征永恒不变，分类学研究更 多的是对物种的鉴定和描述。然而, 生物分类系统 中的界、门、纲、目、科、属、种等各分类等级则
已经携有系统发育和进化的因素。达尔文生物进化 论(Darwin, 1859)为分类学研究带入了进化的思想。 物种不再被认为是一成不变的, 而仅仅是一个独立 的进化单元。

数百年来, 物种概念及物种的划分一直都是生 物分类学中讨论的热点话题。据Mayden (1997)的统 计，广泛使用的物种概念多达 20 余种，如早期就已 经在形态学分类中使用的形态种 (morphospecies)、 数字分类学派的表型种(taxospecies)、古生物学研究

收稿日期: 2016-08-19; 接受日期: 2016-08-27

基金项目: 中国科学院生物多样性委员会物种 2000 中国节点建设项目“菌物物种名录数据库建设”、中国科学院知识创新工程项目(KSCX2-EW-Z-9)、 中国科学院重点部署项目(KSZD-EW-TZ-007)和科技部科技基础性工作专项(2012FY111600)

* 通讯作者 Author for correspondence. E-mail: yaoyj@im.ac.cn 
中的年代种 (chronospecies) 和古生物种 (paleospecies), 以及生物学种(biological species)和系统发育 学种(phylogenetic species)等。这些物种概念可划分 为理论的 (theoretical) 和可操作的 (operational) 两大 类(Mayden, 1997)。理论的物种概念有各种表述, 但 一般都认为: 物种是具有一定形态、生理和生态特 征, 占据一定自然地理区域, 以一定的方式进行繁 衍并相互基因交流的自然生物类群; 并且物种之间 在生殖上相互隔离, 也就是一个物种中的个体一般 不与其他物种的个体交配, 即使交配也不产生有生 殖能力的后代。理论的物种概念除生殖隔离以外, 不包含其他用以区分不同物种的可用标准, 因此在 实际研究中讨论更多的是由此衍生出的可操作的 物种概念, 如形态学种、生物学种和系统发育学种 等等(Taylor et al, 2000)。

\section{1 菌物学中广泛使用的物种概念及其存在 的问题}

\section{1 形态学种}

在菌物学研究中广泛使用的物种概念主要有 形态学种、生物学种和系统发育学种等 3 类。形态 学种(Mayr, 1942)是完全基于形态特征的物种, 其 划分往往具有明确的、可识别的宏观或微观形态依 据, 使用简便。迄今描述的绝大部分菌物物种是以 形态学特征为依据的, 适用于形态学种的概念。以 形态特征为基础建立的分类系统不仅可区分不同 的物种, 在一定程度上也可反映物种的进化历史, 尤其是在一些高阶的分类单元上, 如以有性生殖产 孢结构的差异划分出的接合菌(zygomycetes)、子囊 菌(ascomycetes)、担子菌(basidiomycetes)等。但依 据形态特征进行的传统分类往往无法反映物种间 的进化关系, 特别是在进化末端或不同起源的生物 类群形态特征出现趋同进化现象时。

形态学种概念在分类学研究的早期发挥了不 可替代的作用, 但形态分类学存在很多固有的缺 陷。在菌物分类学研究中, 小型或微型菌物可依据 的形态特征往往十分有限, 不同类群之间极易混 淆, 要发现或描述这些物种都十分困难; 即使是大 型菌物, 形态特征的变异究竟是种内或是种间差异 有时也很难界定。菌物分类中所依据的很多形态特 征如孢子形状、大小、颜色等等, 随着研究标本范 围的扩大, 变异范围往往都由间断的状态逐渐形成
了连续的无法区分的特征, 使可能的物种界限也逐 渐模糊起来。此外, 形态分类不仅要求研究者熟悉 各种形态特征及其变异特点, 而且对物种的生物 学、遗传学、发育学以及种群特征等背景知识也要 有全面的了解, 因此往往只有专业研究人员才能完 成, 尤其是那些小型且复杂的菌物类群。

此外, 尽管形态分类学研究可凭借特征性状的 演变趋势, 以及化石材料等在一定程度上推测出主 要类群的演化历史, 但往往无法为某个进化末端的 类群或单个物种的形成和演化提供更多的证据。

\section{2 生物学种}

与形态学物种概念相比, 生物学物种概念 (Mayr, 1942; Dobzhansky et al, 1977)更接近物种的 本质。它是指一个交配可育的个体群, 种内个体通 过交配和基因交流联系在一起，而与其他物种在生 殖和遗传上存在隔离。生物学物种概念在动物以及 有性繁殖的植物中有较好的应用, 但在菌物分类研 究中的可操作性远远不如形态学种。据估计, 约 $20 \%$ 的菌物不产生减数分裂孢子, 缺乏有性阶段 (Reynolds, 1993)。近年来DNA序列分析发现自然界 中可能还存在大量未描述的菌物物种(Benny et al, 2016), 而这些物种大部分为微小的菌物, 很难观察 到其有性生殖过程, 生物学种概念很难得到应用。 尽管菌物的交配系统及其控制基因在一些模式生 物中有很好的研究, 但菌物的有性生殖过程仍然是 一个谜(Ni et al, 2011)。研究发现, 粟酒裂殖酵母 (Schizosaccharomyces pombe) 等物种中甚至还存在 交配型转变的现象(Heitman et al, 2007)。所有这 些都限制了生物学种概念在菌物分类学研究中的 应用。

\section{3 系统发育学种}

系统发育学种的概念最早由Cracraft (1983)提 出, 与Hennig (1966) 提出的支序物种概念 (cladistic species concept)类似, 是指存在祖裔传承关系的可 鉴别的最小生物群体。系统发育种和支序物种的概 念都强调单系性。在系统发育种概念提出之初, 系 统发育种的划分主要还是依赖于形态特征的衍变 分析, 但近年来随着DNA测序技术的发展, DNA序 列分析已广泛应用于菌物分类和系统发育研究。在 分子系统发育分析中, 单系群成为物种划分的关键 依据。系统发育学种概念与生物学种相比可操作性 更强, 与形态学种相比受主观因素的影响更小, 理 
论上可反映物种的进化历史。但在实际研究中, 系 统发育学种的界定仍然受到诸多因素的限制。早期 菌物的系统发育研究很多都采用ITS单基因分析, 但在部分类群如白僵菌中(Rehner et al, 2011), ITS 的变异水平无法区分近缘种。此外, 单基因系统发 育分析在依据单系群划分物种界限时存在一定的 不确定性。后来, 菌物系统发育研究大多采用多基 因分析的方法，如 $L S U 、 S S U 、 r p b 1 、 r p b 2 、 \beta$-tubulin、 $E F-1 a$ 等都是常用的DNA片段。Taylor等(2000)提出 基于多基因系统发育分析的“谱系一致的系统发育 学种识别法(genealogical concordance phylogenetic species recognition, GCPSR)", 将不同基因在分析中 形成的一致谱系作为物种划分的依据。GCPSR相对 于单基因分析提供了更多的系统发育信息，在很多 形态特征难以区分的类群中如Neurospora (Dettman et al, 2003, 2006)、Fusarium (Starkey et al, 2007)、 Aspergillus (Peterson et al, 2008)等广泛应用。但 GCPSR划分出的物种也需要获得形态、生理、地理 分布、生态分布、寄主范围、生物学特性等方面信 息的支持。近年来随着测序成本的降低, 基因组测 序的菌物物种数量不断增加, 线粒体基因组和核基 因组数据也开始用于菌物的系统发育分析 (Hettiarachchige et al, 2015; Dentinger et al, 2016; Leavitt et al, 2016)。基因组数据理论上能够提供比 多基因序列更多的信息, 但基因组数据如何准确地 反映物种间的系统发育关系，在分析方法上仍然是 一个难题。

\section{2 不同物种概念之间的交错}

形态学种、生物学种及系统发育学种在菌物分 类研究中都有着重要的应用, 但 3 个物种概念出现 冲突的例子并不鲜见。一般来说, 一个形态种有时 会包括不同生物学种和系统发育学种。形态学种与 生物学种冲突的例子以蜜环菌属(Armillaria)的成员 最为典型。由于早期的分类学家以差异甚微的担子 果为物种的主要分类特征, 大部分人认为蜜环菌是 一个单一的物种, 即 Armillaria mellea (Vahl) P. Kumm.。随着该类群交配体系的建立 (Hintikka, 1973; Ullrich \& Anderson, 1978), 蜜环菌被划分为 不同的互交不育群, 大部分不育群此后都找到了形 态、生理和生态上的差异, 而被描述为新的物种 (Korhonen, 1978; Anderson \& Ullrich, 1979)。其他大
型担子菌如粘皮侧耳(Pleurotus ostreatus)等也存在 形态种包含不同生物学种和系统发育学种的实例 (Vilgalys et al, 1993)。而且这种情况在子囊菌、接合 菌及其他小型菌物中可能更为常见。

同样, 生物学种和系统发育学种包含不同形态 学种的例子也在很多类群中存在。比如世界性分布 的红侧耳(Pleurotus djamor (Rumph. ex Fr.) Boedijn) 曾经依据菌盖颜色和光滑度被划分为不同的物种, 但不同菌株的互交和人工栽培实验表明，菌盖颜色 和光滑度更大程度上与生长条件和环境有关, 之前 以此为依据划分出的不同种类实际上为相同的物 种 ${ }^{\circledR}$ 。这种互交实验不仅能帮助人们澄清物种的分 类学问题, 对深入理解分类学所依据的形态变异也 大有裨益。而在系统发育研究中, 某些即使是用多 基因分析也无法区分的物种，却有明显的、稳定的 形态差异。比如广泛栽培的食用菌蟹味菇(也称海鲜 菇、真姬菇等) (Hypsizygus marmoreus (Peck) H.E. Bigelow)就存在白色短柄、褐色短柄、白色长柄、 褐色长柄等不同的形态型，而这些形态型显示的是 栽培性状的差异或是代表着不同物种分化的迹象, 除了增加研究样品的数量、基因片段, 也需要有生 物学实验的支持。

从以上实例我们不难看出, 物种概念尽管在理 论上是明确的, 但物种的划分在实际操作过程中并 没有统一的标准。合理的物种划分需要结合形态、 分子以及生物学等多方面的证据。

\section{3 展望}

目前对生物物种特别是菌物物种的各种界定 和划分都带有一定的探索性，在未来相当长的时间 内可能还是一个不易澄清的问题。但随着技术手段 的发展和认识水平的提高, 对物种的理解将不断深 入, 物种的概念也将越来越清晰。不同的生物类群 往往具有不同的特点, 各种物种概念的应用也会有 不完全相同的含义。也许正是对这些大量错综复杂 的生物类群从不同的角度进行广泛深入的研究, 才 能最终形成适用于地球上所有生物的物种概念。

致谢: 感谢《生物多样性》主编马克平先生和编辑 部的盛情约稿, 并就稿件提供了建设性意见和多方

\footnotetext{
(1) Nicholl D (1996) Relationships within the Pleurotus djamor species complex. Master's thesis. University of Tennessee, Knoxville, TN.
} 
面的帮助。

\section{参考文献}

Anderson JB, Ullrich RC (1979) Biological species of Armillaria mellea in North America. Mycologia, 71, 402-414.

Benny GL, Smith ME, Kirk PM, Tretter ED, White MM (2016) Challenges and future perspectives in the systematics of Kickxellomycotina, Mortierellomycotina, Mucoromycotina, and Zoopagomycotina. In: Biology of Microfungi (ed. Li DW), pp. 65-126. Springer International Publishing, Place.

Cracraft J (1983) Species concepts and speciation analysis. In: Current Ornithology, 1 (ed. Johnston RF), pp. 159-187. Plenum Press, New York.

Darwin CR (1859) On the Origin of Species. John Murray, London.

Dentinger B, Gaya E, O’Brien H, Suz LM, Lachlan R, Díaz-Valderrama JR, Koch RA, Aime MC (2016) Tales from the crypt: genome mining from fungarium specimens improves resolution of the mushroom tree of life. Biological Journal of the Linnean Society, 117, 11-32.

Dettman JR, Jacobson DJ, Taylor JW (2003) A multilocus genealogical approach to phylogenetic species recognition in the model eukaryote Neurospora. Evolution, 57, 2703-2720.

Dettman JR, Jacobson DJ, Taylor JW (2006) Multilocus sequence data reveal extensive phylogenetic species diversity within the Neurospora discreta complex. Mycologia, 98, 436-446.

Dobzhansky T, Ayala FJ, Stebbins GL, Valentine JW (1977) Evolution, pp. 572. W.H. Freeman and Co., San Francisco.

Heitman J, Kronstad JW, Taylor JW, Casselton LA (2007) Sex in Fungi: Molecular Determination and Evolutionary Implications. ASM Press, Washington, DC.

Hennig W (1966) Phylogenetic Systematics. University of Illinois Press, Illinois.

Hettiarachchige IK, Ekanayake PN, Mann RC, Guthridge KM, Sawbridge TI, Spangenberg GC, Forster JW (2015) Phylogenomics of asexual Epichloë fungal endophytes forming associations with perennial ryegrass. BMC Evolutionary Biology, 15, 27.

Hintikka V (1973) A note on the polarity of Armillaria mellea. Karstenia, 13, 32-39.

Hong DY (2016a) Opinion of raising rationality in species delimitation. Biodiversity Science, 24, 360-361. (in Chinese) [洪德元 (2016) 关于提高物种划分合理性的意见. 生物多样性, 24, 360-361.]

Hong DY (2016b) Biodiversity pursuits need a scientific and operative species concept. Biodiversity Science, 24, 979 -999. (in Chinese with English abstract) [洪德元 (2016) 生
物多样性事业需要科学、可操作的物种概念. 生物多样 性, 24, 979-999.]

Korhonen K (1978) Intersterility and clonal size in the Armillaria mellea complex. Karstenia, 18, 31-42.

Leavitt SD, Grewe F, Widhelm T, Muggia L, Wray B, Lumbsch HT (2016) Resolving evolutionary relationships in lichen-forming fungi using diverse phylogenomic datasets and analytical approaches. Scientific Reports, 6, 22262.

Linnaeus C (1753) Species Plantarum, Tomus 2. Laurentius Salvius, Stockholm, Sweden.

Mayden RL (1997) A hierarchy of species concepts: the denouement in the saga of the species problem. In: Species: The Units of Biodiversity (eds Claridge MF, Dawah HA, Wilson MR), pp. 381-424. Chapman \& Hall, London.

Mayr E (1942) Systematics and the Origin of Species. Columbia University Press, New York.

Ni M, Feretzaki M, Sun S, Wang X, Heitman J (2011) Sex in fungi. Annual Review of Genetics, 45, 405.

Peterson SW (2008) Phylogenetic analysis of Aspergillus species using DNA sequences from four loci. Mycologia, 100, 205-226.

Rehner SA, Minnis AM, Sung GH, Luangsa-ard JJ, Devotto L, Humber RA (2011) Phylogeny and systematics of the anamorphic, entomopathogenic genus Beauveria. Mycologia, 103, 1055-1073.

Reynolds DR (1993) The fungal holomorph: an overview. In: The Fungal Holomorph: Mitotic, Meiotic and Pleomorphic Speciation in Fungal Systematics (eds Reynolds DR, Taylor JW), pp. 15-25. CAB International, Wallingford, UK.

Starkey DE, Ward TJ, Aoki T, Gale LR, Kistler HC, Geiser DM, Suga H, Tóth B, Varga J, O’Donnell K (2007) Global molecular surveillance reveals novel Fusarium head blight species and trichothecene toxin diversity. Fungal Genetics and Biology, 44, 1191-1204.

Taylor JW, Jacobson DJ, Kroken S, Kasuga T, Geiser DM, Hibbett DS, Fisher MC (2000) Phylogenetic species recognition and species concepts in fungi. Fungal Genetics and Biology, 31, 21-32.

Ullrich RC, Anderson JB (1978) Sex and diploidy in Armillaria mellea. Experimental Mycology, 2, 119-129.

Vilgalys R, Smith A, Sun BL, Miller OK (1993) Intersterility groups in the Pleurotus ostreatus complex from the continental United States and adjacent Canada. Canadian Journal of Botany, 71, 113-128. 